\title{
Die Schilddrüse der alten Katze - Was gibt es Neues in der Diagnostik?
}

Ruth Klein

Die Zeit der Bremer Stadtmusikanten ist vorbei, man trennt sich heutzutage nicht mehr von Hund und Katze, nur, weil sie nutzlos geworden sind. So können Katzen bei uns alt werden. Mit zunehmendem Alter wechseln bisher subklinische Erkrankungen der Organsysteme zum klinisch erkennbaren Stadium. Zu den typischen Problemen der alten Katze gehört neben renalen und kardialen Erkrankungen und dem Diabetes mellitus auch die Hyperthyreose.

Dies war Anlass für die American Association of Feline Practitioners 2016 die „Guidelines for the Management of Feline Hyperthyroidism“ heraus zu geben, um dem Praktiker eine Hilfestellung in der Diagnose und Therapie zu geben [1]. Aber welche Auswirkung hat ein nicht dem Bedarf entsprechendes Überangebot der Schilddrüsenhormone auf die Organsysteme, die altersbedingt aus anderen Gründen bereits vorgeschädigt sein können und wie kann ein Laborbefund hilfreich sein?

\section{Pathogenese}

Pathohistologisch sind gutartige Neoplasien (Adenom) und adenomatöse Hyperplasien sowie bei ca. $2 \%$ der Katzen mit Schilddrüsenüberfunktion ein Karzinom zu finden [8]. Bei der felinen Hyperthyreose ist aber bis jetzt keine ursächliche Noxe beschrieben.

Häufig werden die veränderten Lebensumstände der Katze diskutiert v.a. die Fütterung mit kommerziellem Futter in Verbindung mit erhöhter Jodaufnahme, aber auch die Aufnahme von PCBs (polychlorierten Biphenylen).

\section{Häufigkeit}

Die Auswertung der bei Laboklin gemessenen T4-Konzentrationen eines $1 / 2$ Jahres zeigen, dass der Prozentsatz älterer
Katzen mit erhöhten Werten im 2-stelligen Prozentbereich liegt ( $\triangleright$ Abb. 1).

\section{Symptomatik und Verände- rung der Laborwerte}

\section{T4 und T3}

90\% der sezernierten Schilddrüsenhormone ist T4. T4 ist primär eine proteingebundene zirkulierende Reserve der Schilddrüsenhormone im Blut. In Leber, Nieren, Herz und Hypothalamus wird T4 zu T3 deiodiniert und so in die aktive Form transformiert.

\section{T3 ist für die Schilddrüsenhormon- wirkung verantwortlich.}

Physiologisch erhöhen die Schilddrüsenhormone den Grundumsatz. Dies zeigt sich in:

- erhöhtem Sauerstoffverbrauch,

- erhöhtem Substratumsatz

- gesteigerter Energiegewinnung

- erhöhter Thermogenese

\section{Stoffwechselwirkung}

T3 aktiviert im Fettstoffwechsel die Lipolyse, den Auf- und Abbau von Cholesterin und beschleunigt die Freisetzung von Glycerin und freien Fettsäuren. Im Kohlenhydratstoffwechsel stimuliert T3 die hepatische Glukoneogenese, den Glykogenabbau und fördert intestinal die Glukoseresorption. Gleichzeitig hemmt T3 die Speicherung von Kohlenhydraten. Katzen sind aufgrund ihrer Nahrungszusammensetzung mit einem geringen Kohlenhydratanteil enzymatisch auf einen ausgeprägten Proteinumsatz ausgelegt, hier spielen die Schilddrüsenhormone im Kohlenhydratstoffwechsel eine entscheidende Rolle.

Bei steigenden Hormonkonzentrationen zeigen viele Katzen Polyphagie. Eine Hypermoltilität des Darms - T3 stimuliert die Darmmotilität durch Aktivierung der glatten Muskulatur - zeigt sich in frequenterem Kotabsatz mit breiiger Konsistenz. Zusammen mit der Wirkung auf den Glukose- und Fettstoffwechsel ist ein Abmagern trotz gesteigerter Futteraufnahme die Folge. Es kommt aber nicht nur zum Abbau der Fettdepots. Bei der Hyperthyreose ist die Schilddrüsenhormonwirkung im Protein- und Muskelstoffwechsel über die gesteigerte Gluconeogenese aus glukoplastischen Aminosäuren katabol [4], es kommt zur Re-

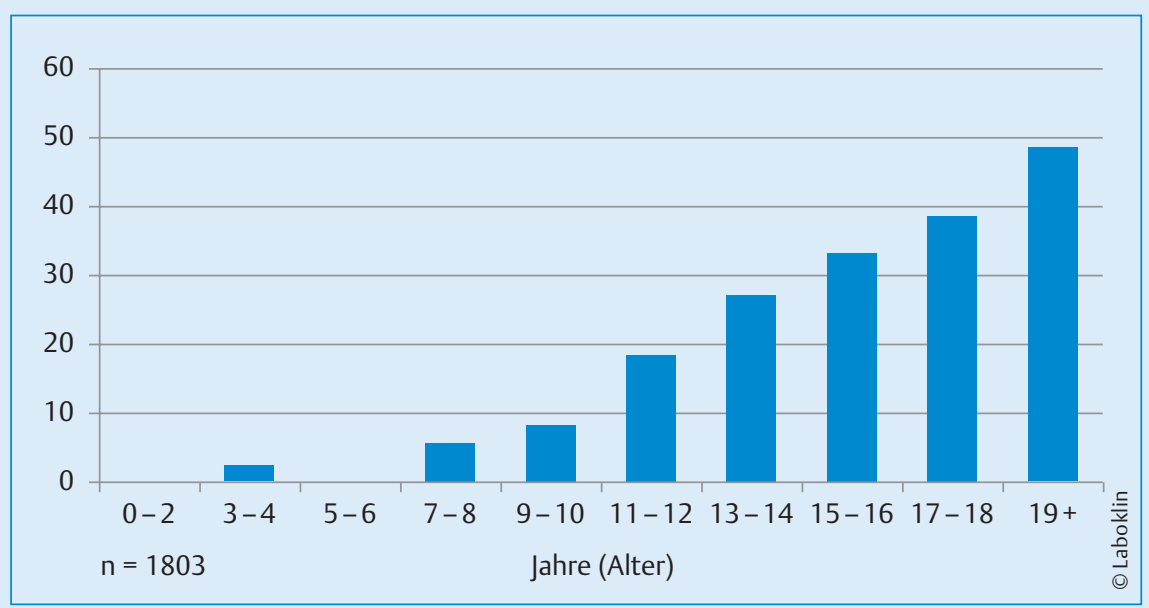

Abb. 1 Auswertung der gemessenen T4-Konzentrationen bei Katzen $(n=1803)$ : der Prozentsatz an Tieren mit erhöhten Schilddrüsenwerten liegt bei Katzen > 10 Jahren im 2-stelligen Prozentbereich. 
duktion der Muskelmasse, die Tiere wirken mager. Aufgrund der glukoplastischen Wirkung bleibt die Glukosekonzentration trotz erhöhten Bedarfs im Normbereich. Durch den erhöhten Proteinumsatz sinken auch die Fruktosamin- $[3,5]$ und Albuminkonzentrationen ab. Eine Beurteilung des Langzeitblutzuckerspiegels ist bei diesen Patienten somit nicht möglich.

Die Kreislaufwirkung von T3 ist vielfältig, kardial vor allem positiv inotrop und chronotrop. Peripher sinkt der Gefäßwiderstand. Bei der Hyperthyreose erscheinen die Katzen häufig aktiver und munterer, was von den Besitzern eher positiv gesehen wird. Viele Patienten sind aber unruhig, hyperaktiv, auskultatorisch tachykard und zeigen Hypervokalisation. Der systolische Blutdruck steigt bei sinkendem diastolischem Blutdruck. Diese Dauerbelastung des Myokards führt zu einem Anstieg der cTroponin-I- und pro-BNP-Konzentration [2,6]. cTroponin-I ist bei erhöhten Blutkonzentrationen ein sensibler Marker für die kardiale Schädigung.

Polyurie und Polydypsie sind häufige Symptome der Hyperthyreose. Mit steigendem Blutdruck kommt es renal zur Hyperfiltration mit steigender glomerulärer Filtrationsrate bei sinkender tubulärer Rückresorption. Der Laborbefund ist gekennzeichnet durch unauffällige Harnstoff- und Kreatininkonzentratio- nen [7]. Bei folgender Schädigung der Glomerula kommt es zur Proteinurie. Steigende BNP-Konzentrationen führen zur forcierten Natriurese.

Viele Katzen wirken struppig, da sie sich nicht mehr pflegen, aber auch, weil der Haarzyklus beschleunigt ist. Die Haare werden dünner und das Haarkleid wirkt licht.

Über den direkten Einfluss der Schilddrüse auf die Thermoregulation steigt die Körpertemperatur subfebril.

Ein hoher Prozentsatz der Katzen > 9 Jahre leidet an einer Organvorschädigung, wie z.B. einer reduzierten Leberleistung. Vorgeschädigte Organe reagieren nicht immer adäquat. So ist Polyphagie nicht das Kardinalsymptom bei allen Katzen, nicht alle sind hyperaktiv oder zeigen PU/PD.

\section{Differenzialdiagnosen und Folgeerkrankungen}

Klassische Differenzialdiagnosen sind je nach Symptomatik Pankreasinsuffizienz, Diabetes mellitus, Kardiomyopathien, Enteropathien, Nephropathie und Tumorosen.

Die Hyperthyreose kann massive Schäden an den Organen verursachen. Kardiomyopathien, Nierenschädigung, Pankreatitiden und Hepatopathien sind häu- konret

Einige hyperthyreote Katzen sind anorektisch, auffallend ruhig und zeigen ein normales Harn- und Kotabsatzverhalten - trotz Fehlens der typischen Symptomatik kann eine Hyperthyreose vorliegen.

fige Folgeerkrankungen. Erstere sind zumeist durch den erhöhten Blutdruck bedingt, letztere durch die forcierte Stoffwechselleistung der Leber.

\section{Labordiagnostik}

Neben der Klinik gibt der Laborbefund erste Hinweise oder sichert über die Bestimmung der Schilddrüsenhormone T4, TSH und bei grenzwertigen Ergebnissen auch fT4, die Diagnose. Die Bestimmung der T3-Konzentration ist möglich, aber eine moderate Erhöhung kann ebenso durch den Transport und die Aufregung bedingt sein.

Labordiagnostisch sollte aufgrund der gravierenden Auswirkungen der Hyperthyreose auf die Organsysteme ein klinisch-chemisches Screening durchgeführt werden. Mit den Ergebnissen können mögliche Differenzialdiagnosen ausgeschlossen werden. ProBNP, cTroponin-I sowie Harnstoff und Kreatinin dienen nicht der Diagnose selbst, sind aber

Tab. 1 Bei einem Verdacht auf eine Hyperthyreose zu bestimmende Blutparameter inklusive möglicher Veränderungen und Differenzialdiagnosen.

\begin{tabular}{|c|c|c|c|}
\hline Organsystem & Parameter & $\begin{array}{l}\text { Veränderungen bei } \\
\text { Hyperthyreose }\end{array}$ & Differenzialdiagnose \\
\hline Schilddrüse & $\begin{array}{l}\text { T4 } \\
\text { TSH } \\
\text { T3 } \\
\text { fT4 }\end{array}$ & $\begin{array}{l}\text { erhöht } \\
\text { erniedrigt } \\
\text { erhöht oder normal } \\
\text { erhöht }\end{array}$ & \\
\hline Leber & $\begin{array}{l}\text { ALT, GLDH, AP, } \\
\text { Gesamteiweiß und Albumin }\end{array}$ & $\begin{array}{l}\text { erhöht } \\
\text { erniedrigt }\end{array}$ & \\
\hline Nieren & $\begin{array}{l}\text { Harnstoff, Kreatinin, SDMA } \\
\text { (symetrisches Dimethylarginin), } \\
\mathrm{Na}, \mathrm{K}, \mathrm{Ca} \text { und PO4 }\end{array}$ & $\begin{array}{l}\text { niedrig normal } \\
\text { Kontrolle }\end{array}$ & erhöht bei Niereninsuffizienz \\
\hline Herz/Kreislauf & pro-BNP und cTroponin I & erhöht & \\
\hline Pankreas & $\begin{array}{l}\text { pankreatische Lipase, } \\
\text { TLI }\end{array}$ & erhöht & TLI: erniedrigt bei Pankreasinsuffizienz \\
\hline Kohlenhydratstoffwechsel & Fruktosamin, Glukose & erniedrigt & erhöht bei Diabetes \\
\hline
\end{tabular}


wichtige Parameter in der Beurteilung der Schwere der Erkrankung und den Spätfolgen trotz Therapie.

\section{Therapie}

Mit der Therapie sollte umgehend nach der Diagnosestellung begonnen werden, um weitere Schäden an den Organen zu verhindern. Welche Art der Medikation Thyreostatika, Radiojodtherapie oder jodfreies Futter - gewählt wird, ist abhängig vom Patienten, dem Alter, dem Erkrankungsstadium und der Compliance der Besitzer. Letztendlich ist es auch immer eine finanzielle Entscheidung.

\section{Therapiekontrolle - und was bleibt übrig?}

Die erste Therapiekontrolle sollte nach 2-4 Wochen erfolgen und neben der klinischen Untersuchung und der Messung der Schilddrüsenhormone auch immer eine Messung der bereits erwähnten Parameter beinhalten. Dies ermöglicht eine objektive Beurteilung der Organsituation. Normalisieren sich unter der Therapie die vorher veränderten Parameter nicht, besteht erweiterter Therapiebedarf.

Anders bei den Nierenparametern: steigen die Werte unter Therapie deutlich an oder überschreiten den Referenzbereich, liegt eine durch die Hyperthyreose kaschierte Niereninsuffizienz vor, die entsprechend therapiert werden muss.

\section{.konkret}

Bei einer durch die Hyperthyreose kaschierten Niereninsuffizienz ist es keine Option, die Schilddrüsentherapie zu reduzieren, um die Nierenwerte wieder in die Norm zu bekommen.

Eine weitere Schädigung der Niere mit Ausfall weiterer Glomerula wäre die Folge und es droht auf Dauer ein Nierenversagen. Die spezifische Nierentherapie ist daher unumgänglich.

Online zu finden unter

http://dx.doi.org/10.1055/s-0043-102345

\section{Literatur}

1 Carney HC, Ward CR, Bailey SJ et al. 2016 AAFP Guidelines for the Management of Feline Hyperthyroidism. J Feline Med Surg 2016; 18 (5): 400-416

2 Connolly DJ, Guitian J, Boswood A et al. Serum troponin I levels in hyperthyroid cats before and after treatment with radioactive iodine. J Feline Med Surg 2005; 7 (5): 289-300

3 Graham PA, Mooney CT, Murray M. Serum fructosamine concentrations in hyperthyroid cats. Res Vet Sci 1999; 67 (2): 171-175

4 Peterson ME, Castellano CA, Rishniw M. Evaluation of Body Weight, Body Condition, and Muscle Condition in Cats with Hyperthyroidism. J Vet Intern Med 2016; 30 (6): 1780-1789

5 Reusch CE, Tomsa K. Serum fructosamine concentration in cats with overt hyperthyroidism. J Am Vet Med Assoc 1999; 215 (9): 1297-1300
6 Sangster JK, Panciera DL, Abbott JA et al. Cardiac biomarkers in hyperthyroid cats. J Vet Intern Med 2014; 28 (2): 465-472

7 Vaske HH, Schermerhorn T, Grauer GF. Effects of feline hyperthyroidism on kidney function: a review.] Feline Med Surg 2016; 18 (2): 55-59

8 Wehner A. Besitzerinformation: Schilddrüsenüberfunktion (Hyperthyreose) bei der Katze. Im Internet: http://www.med.vetmed. uni-muenchen.de/einrichtungen/innere_ medizin/leistungen/endokrinologie/ hyperthyreose.pdf Datum des Zugriffs: 26.01.2017

\section{Dr. Ruth Klein}

Laboklin GmbH

Steubenstr. 4

97688 Bad Kissingen

klein@laboklin.com 